\title{
Avaliação da atenção básica pela perspectiva dos usuários: adaptação do instrumento EUROPEP para grandes centros urbanos brasileiros
}

\author{
Evaluation of primary care from the perspective of users: \\ adaptation of the EUROPEP instrument \\ for major Brazilian urban centers
}

\author{
Ana Laura da Rocha Bastos da Silva Brandão ${ }^{1}$ \\ Ligia Giovanella ${ }^{1}$ \\ Carlos Eduardo Aguilera Campos ${ }^{2}$
}

${ }^{1}$ Departamento de Administração e Planejamento em Saúde, Escola Nacional de Saúde Pública, Fundação Oswaldo Cruz. Av. Brasil 4036/1001, Manguinhos. 21040-361 Rio de Janeiro RJ. alaurabrandao@gmail.com ${ }^{2}$ Programa de Atenção Primária à Saúde, Faculdade de Medicina, Universidade Federal do Rio de Janeiro.

\begin{abstract}
Satisfaction with health care is a multidimensional concept that considers aspects such as access, organization and professional-user interaction. The aim of this study was to adapt and apply an instrument in the Family Health Strategy (FHS) to assess user satisfaction with Primary Health Care (PHC) based on the European Task Force on Patient Evaluation of General Practice Care (EUROPEP), which refers to user satisfaction wth general and family medicine services. The instrument consists of five dimensions of satisfaction: relationship and communication, medical care, information and support, continuity and cooperation, and organization of services. The study was divided into phases: review and adjustment of the instrument and application to a representative sample of users of the FHS in Rio de Janeiro. The averages of the proportions of answers for each indicator were calculated to analyze the results. Relationship and communication between professionals and users received the best evaluation and Organization of Services eceived the worst appraisal. Regarding education level, good self-perceived health and more elderly were more satisfied. The instrument proved to be easy to apply, can be routinely used for monitoring of the FHS, and is a tool for the institutionalization of evaluation.
\end{abstract}

Key words Satisfaction, Evaluation, Primary health care
Resumo A satisfação com cuidados de saúde é conceito multidimensional que considera aspectos como acesso, organização, interação usuárioprofissional. O objetivo do estudo foi adaptar e aplicar na Estratégia de Saúde da Família (ESF) um instrumento de avaliação da satisfação do usuário em Atenção Primária à Saúde (APS), tendo por base o European Task Force on Patient Evaluation of General Practice Care (EUROPEP), que refere-se à satisfação dos usuários de serviços de medicina geral e familiar (composto por cinco dimensões de satisfação: relação e comunicação, cuidados médicos, informação e apoio, continuidade e cooperação, e organização dos serviços). $O$ estudo foi composto por fases: revisão, adaptação do instrumento e aplicação a uma amostra representativa dos usuários da ESF no município do Rio de Janeiro. Para análise dos resultados foram calculadas médias das proporções de respostas para cada indicador, sendo Relação e Comunicação entre profissional e usuário o que mais se destacou e o de Organização dos Serviços recebeu a pior avaliação. Em relação à escolaridade, boa autopercepção de saúde e mais idosos mostraramse mais satisfeitos. O instrumento mostrou ser de fácil aplicação, podendo ser utilizado rotineiramente para monitoramento da ESF, sendo importante ferramenta para a institucionalização da avaliação.

Palavras-chave Satisfação, Avaliação, Atenção Primária à Saúde 


\section{Introdução}

No Brasil, durante a década de 1990, a atenção básica $(\mathrm{AB})$ obteve destaque, com a introdução de programas inovadores com objetivos de realizar mudanças no modelo assistencial como o Programa de Agentes Comunitários de Saúde e o Programa de Saúde da Família ${ }^{1}$. A grande expansão da Estratégia Saúde da Família (ESF) trouxe a necessidade de qualificação da atenção básica ofertada à população, tornando necessário o desenvolvimento e a implementação de ferramentas que permitissem a avaliação da implantação da Estratégia de forma permanente, para orientar com agilidade o processo decisório, e melhorar os resultados para a população.

Muitas iniciativas com foco na avaliação da ESF vêm sendo desenvolvidas no Brasil desde a década de 1990. Apesar de pesquisas incentivadas pelo Ministério da Saúde para avaliações dos serviços de saúde para nortear a elaboração e a reorientação de políticas e programas na $\mathrm{AB}^{2}$, a avaliação da ESF desde a perspectiva dos usuários ainda é escassa e não estão disponíveis instrumentos de fácil aplicação para uso rotineiro.

Estudos de satisfação podem contribuir para a institucionalização da avaliação e reorganização dos serviços além de ser uma ferramenta para a participação popular.

A satisfação é um processo dinâmico que pode ser influenciado por uma série de fatores como percepção do estado de saúde e da doença, crenças, características sociodemográficas e envolve diversos aspectos de atenção. Por conta desse conceito multidimensional, a satisfação com a atenção à saúde pode ser caracterizada como uma avaliação individual de várias dimensões dos cuidados de saúde, tais como: acesso, infraestrutura, interação usuário-profissional, e resultados em termos de saúde ${ }^{3-5}$.

Os estudos de satisfação no campo da saúde se sobressaem na literatura na década de 1970 com destaque para os Estados Unidos e Inglaterra inseridos segundo Esperidião e $\operatorname{Trad}^{6}$ no paradigma de consumismo e da cultura da qualidade e tinham como objetivo conseguir melhores resultados clínicos, por meio da adesão ao tratamento.

No Brasil, os estudos de satisfação passa a ser mais difundidos na década de 1990, a partir da discussão do conceito de accountability (prestação de contas), juntamente com o fortalecimento da participação da comunidade nas decisões, nos processos de avaliação e planejamento ${ }^{3}$.

O conceito de satisfação se relaciona com o de responsividade, este último desenvolvido pela
Organização Mundial da Saúde (OMS) e difundido no relatório sobre a avaliação do desempenho dos sistemas de saúde de 2000. As diferenças pontuadas por $^{7}$ entre responsividade e satisfação dos pacientes estão centradas em três pontos: i) responsividade avalia o sistema de saúde como um todo e a satisfação dos pacientes concentra-se na interação dos cuidados clínicos; ii) a responsividade está relacionada aos aspectos não médicos e a satisfação dos pacientes aos aspectos médicos e não médicos; e iii) a responsividade avalia as percepções individuais e a satisfação dos pacientes representa uma mistura complexa das necessidades percebidas com as expectativas individuais e a experiência do cuidado.

A satisfação dos usuários tem ocupado um lugar progressivamente mais importante na avaliação da qualidade dos serviços. Esta posição considera que a satisfação está diretamente relacionada à adesão terapêutica e com os resultados dos cuidados em saúde, influenciando comportamentos de saúde e doença, além da crescente valorização do papel do usuário enquanto consumidor ${ }^{8}$.

Diversos autores mostram a influência da satisfação na adesão ao tratamento, que é um processo que exige a construção de vínculos e das condições que possam consolidar uma boa relação paciente profissional/equipe de saúde $e^{4,9,10}$. O estudo de revisão ${ }^{6}$ em 56 artigos publicados em revistas indexadas pelo MEDLINE e Web of Science, de 1970 a 2005, encontrou que usuários satisfeitos tendem a aderir ao tratamento prescrito, fornecem informações importantes para o prestador, e ainda, que os pacientes satisfeitos são mais propensos a ter melhor qualidade de vida.

Buscando contribuir para o desenvolvimento de metodologia de avaliação o objetivo do presente estudo foi adaptar e aplicar um instrumento de avaliação da satisfação na perspectiva do usuário, para a ESF, tendo por base do modelo europeu de avaliação da qualidade em APS o European Task Force on Patient Evaluation of General Practice Care (EUROPEP). É um instrumento utilizado internacionalmente e refere-se à satisfação dos usuários de serviços médicos de medicina geral e familiar na Europa.

\section{Métodos}

O questionário EUROPEP começou a ser desenvolvido em 1995 por pesquisadores de oito países e sua validação finalizou em 1998, após passar por diversas fases de elaboração: estudo de 
prioridades dos usuários e desenvolvimento do instrumento (1996), estudo piloto qualitativo (1996); estudo piloto quantitativo (1996); procedimento formal de tradução (1997); estudo de validação (1997); seleção final dos itens (1997); e validação final: (1998) e posteriormente foi aplicado em 16 países ${ }^{11}$.

O EUROPEP foi projetado para fornecer feedback para a melhoria da prática, desempenho e organização dos cuidados dos profissionais médicos de família ${ }^{11}$.

No presente estudo o instrumento foi adaptado e aplicado a uma amostra de usuários de ESF em um Centro de Saúde Escola no município do Rio de Janeiro.

A adaptação do instrumento, para a realidade no Brasil, considerou três etapas. A primeira foi a tradução do questionário do português de Portugal para o português do Brasil. A seguir o questionário foi submetido a três reuniões, com profissionais da ESF, usuários e ACS com objetivo verificar se os processos avaliados no instrumento correspondiam às ações e à organização do trabalho da ESF, além de envolver os profissionais no processo de avaliação.

O questionário foi revisto considerando as sugestões apresentadas, seguido da realização de pré-teste. Nesta terceira fase do processo de adaptação o instrumento foi aplicado a um número reduzido de pessoas cadastradas (30) da USF. Nessa fase foram examinadas a adequabilidade do instrumento à realidade, a forma e tempo de aplicação, as dúvidas e questões dos entrevistadores e dos usuários no momento da entrevista e a forma mais adequada para abordagem aos usuários. Procedeu-se a seguir à nova revisão do instrumento para aplicação a amostra representativa.

O instrumento utilizado é constituído por 3 partes: i) Indicadores chaves (relação e comunicação, cuidados médicos, informação e apoio, continuidade e cooperação, e, organização dos serviços); ii) Indicadores de áreas específicas de satisfação (consulta, marcação e acessibilidade, características dos profissionais, condições do centro de saúde e os serviços prestados); iii) Informações sobre os usuários (dados socioeconômicos, de saúde e, atitudes após a experiência).

Os critérios de elegibilidade dos sujeitos para a aplicação do questionário foram ser usuário há mais de um ano da equipe de saúde da família, ser maior de 18 anos, ter tido consulta com profissional médico, ou ser acompanhante de menores de idade em consulta médica no dia da entrevista.

Como se pretendeu avaliar a atuação do médico de Saúde da Família o cálculo da amostra levou em consideração o número de consultas médicas por mês de cada equipe de saúde da família no ano de 2009. Foi calculada uma amostra de 288 pacientes, com margem de erro de $6 \%$ e intervalos de confiança de 95\%. Foram aplicados questionários a 289 usuários no período de junho a agosto de 2010.

A aplicação do questionário levou em média 15 minutos, ou seja, podemos considerá-lo um instrumento de rápida aplicação.

Para mensuração das respostas das perguntas fechadas foi utilizada uma escala, que aborda a satisfação pela concordância ou discordância com as afirmativas sobre o último atendimento, sobre o serviço e sobre o profissional, a partir de categorias representadas por figuras de faces em cinco expressões distintas de satisfação (1- muito bom; 2- bom; 3-regular; 4- ruim, 5- péssimo e 6- não se aplica).

Para análise dos resultados foram calculadas médias das proporções das respostas "muito bom" e "bom" para cada indicador chave, e com esses valores foi possível obter o índice de satisfação global.

Os indicadores chave e as perguntas correspondentes a cada indicador são apresentadas no Quadro 1.

$\mathrm{Na}$ análise final, da satisfação dos usuários proposta pelo EUROPEP, os resultados de cada indicador chave são classificados como muito bom, bom, regular, ruim e péssimo por meio da média das respostas de muito bom e bom de cada indicador.

A valoração geral de cada um dos Indicadores Chave foi obtida somando-se as respostas "muito bom" e "bom" e dividindo pelo número de perguntas referentes a cada indicador. O indicador é classificado como "muito bom" se a média das proporções de respostas muito bom e bom ultrapassam 85\%, "bom" é considerado entre 84\% a $65 \%$, "regular" entre $64 \%$ a $45 \%$, "ruim" entre 44\% a 25\% e "péssimo" entre 24\% e 0\%.

Para verificar possíveis associações entre os padrões de resposta e as características dos usuários, foram realizadas análises relacionando as variáveis com o perfil dos pacientes. Para isso, a amostra total foi analisada segundo determinadas características ponderadas como importantes na literatura para esse tipo de análise, uma vez que estas podem influenciar os níveis de satisfação dos usuários. As características eleitas para realizar tal comparação entre respostas foram: sexo; nível de escolaridade; autopercepção de saúde e faixa etária. Neste sentido, foi realizado um teste de hipótese onde a hipótese nula é de que a diferença entre 
estas duas proporções é zero. Assim, quando refutada a hipótese nula, significa que há diferença na proporção de satisfeitos entre as categorias. Para isso, foi utilizado o pacote estatístico STATA 10.

Ao final da entrevista o usuário foi convidado a expressar opiniões e sugestões através da pergunta aberta: "Diga, por favor, o que precisa ser melhorado no Centro de Saúde”. Dos 289 questionários aplicados, 176 (61\%) usuários fizeram considerações a respeito de melhoria no centro de saúde.

As respostas foram desmembradas em 254 menções, já que em uma mesma resposta os usuários abordavam diversos temas e foram categorizadas e agrupadas conforme conteúdo similar, em 20 grandes categorias. Ao final foram contabilizadas as respostas de acordo com cada categoria de análise.

\section{Resultados}

Os usuários entrevistados eram em sua maioria mulheres (84\%), com faixa média de 30 a 39 anos e baixa escolaridade (53\% não possuíam o ensino elementar completo). Na autopercepção de saúde, $40 \%$ dos entrevistados indicaram saúde regular.

O Gráfico 1 apresenta os resultados da avaliação dos usuários por indicadores chave.

$\mathrm{O}$ indicador Relação e Comunicação foi o que mais se destacou positivamente em comparação aos outros indicadores. Dentre as perguntas que o compõem, a que obteve a maior gradação de resposta "muito bom", foi o interesse do profissional sobre o usuário (39\%). Outro ponto de elevada satisfação dos usuários foi a variável de sentir-se a vontade com o médico (38\%). Ambas as questões são fundamentais para o estabelecimento de boa relação usuário profissionais no momento da atenção à saúde (Gráfico 1).

O Indicador Cuidados Médicos apresentou o segundo melhor resultado. O item que mais se destacou positivamente, com a maior gradação de respostas "muito bom" foi a qualidade do exame físico realizado pelo profissional (37\%) (Gráfico 1).

No Indicador Informação e Apoio os itens que mais se destacaram positivamente, com gra-

Quadro 1. Indicadores chave e variáveis para avaliação da satisfação, do instrumento Europep

\begin{tabular}{|l|l|}
\hline Indicadores chave & \multicolumn{1}{c|}{ Variáveis } \\
\hline Relação e & $\begin{array}{l}\text { Duração do tempo da consulta } \\
\text { Interesse do profissional nos seus problemas de saúde e a forma como ele o ouviu } \\
\text { Sentiu-se à vontade para contar os seus problemas ao profissional } \\
\text { Sua participação nas decisões do cuidado que o profissional prestou } \\
\text { Segredo/sigilo da informação sobre o seu caso }\end{array}$ \\
\hline Cuidados Médicos & $\begin{array}{l}\text { Alívio rápido dos seus sintomas } \\
\text { Sentiu melhora para desempenhar suas tarefas diárias } \\
\text { Qualidade do exame físico que o profissional fez durante a consulta } \\
\text { Indicação de serviços de prevenção de doenças como, por exemplo, exames e vacinas }\end{array}$ \\
\hline $\begin{array}{l}\text { Informação e } \\
\text { Apoio }\end{array}$ & $\begin{array}{l}\text { Explicação sobre os exames e tratamentos prescritos } \\
\text { Informações sobre o que gostaria de saber dos seus sintomas e da sua doença } \\
\text { Profissional abordou questões emocionais relacionados a seus problemas de saúde } \\
\text { Incentivo fornecido pelo profissional para compreensão da importância de seguir as } \\
\text { orientações e tratamento de saúde }\end{array}$ \\
\hline $\begin{array}{l}\text { Continuidade e } \\
\text { Cooperação }\end{array}$ & $\begin{array}{l}\text { Conhecimento do profissional sobre ele e o seu caso } \\
\text { Preparação sobre o que esperar de especialistas e dos cuidados hospitalares quando } \\
\text { necessário }\end{array}$ \\
\hline $\begin{array}{l}\text { Organização dos } \\
\text { serviços }\end{array}$ & $\begin{array}{l}\text { Apoio dos profissionais do Centro de Saúde, além daquele que o atendeu } \\
\text { Facilidade em marcar uma consulta em horário e data adequada no Centro de Saúde } \\
\text { Tempo de espera para ser atendido } \\
\text { Rapidez com que os problemas urgentes foram resolvidos }\end{array}$ \\
\hline
\end{tabular}

Fonte: Elaboração própria 
Relação e Comunicação

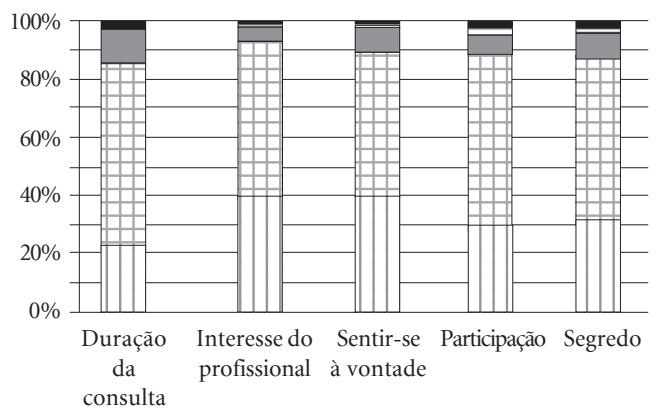

Organização do Serviço

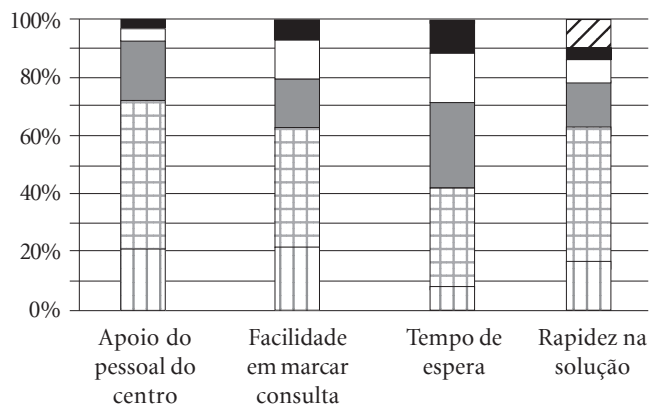

Informação e Apoio

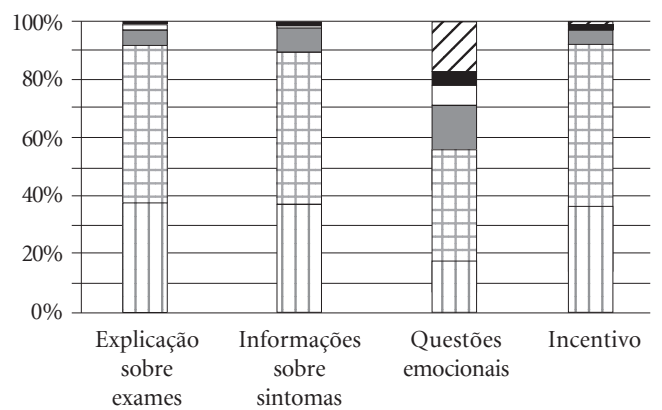

Cuidados Médicos

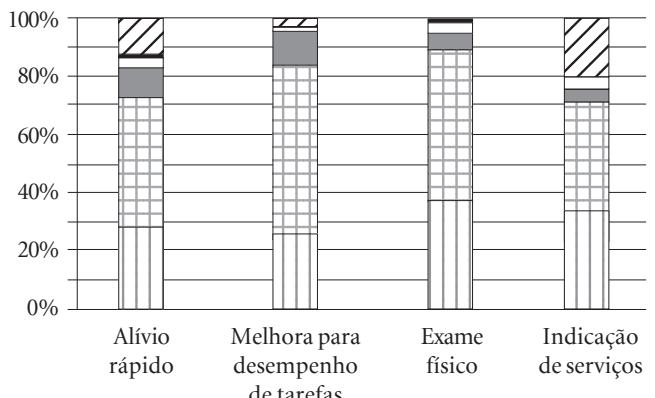

Continuidade e Cooperação

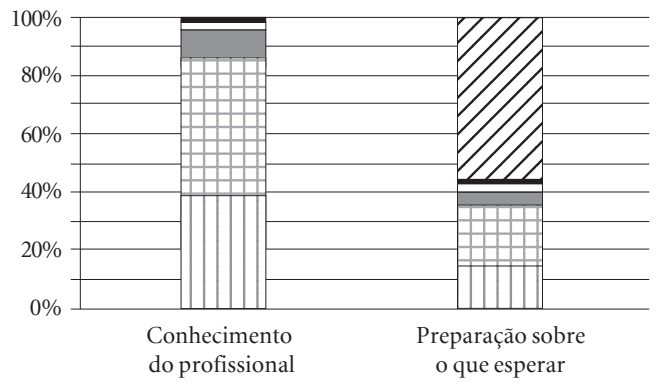

Gráfico 1. Avaliação dos Indicadores Chaves e componentes de satisfação por usuários da ESF, Centro de Saúde Escola, Rio de Janeiro, 2010.

dação elevada de respostas "muito bom", foi a explicação do profissional sobre exames (38\%), e informações sobre os sintomas da doença $(37 \%)$. Neste indicador a pior avaliação, com maior proporção de respostas "péssimo" e "ruim" foi para o critério de abordagem dos profissionais médicos sobre as questões emocionais (12\%). Somando-se aos 18\% que responderam "não se aplica”, este resultado, mostra que é uma questão importante para a qualidade do atendimen- to, a abordagem de questões emocionais e psicossociais do indivíduo nas consultas médicas está pouco presente no cuidado (Gráfico 1).

No Indicador Continuidade e Cooperação o conhecimento do profissional médico sobre o usuário e o seu caso foi considerado "Muito bom" por $38 \%$ e "bom” para $48 \%$ dos usuários (Gráfico 1$)$.

O Indicador Organização dos serviços foi o que recebeu a pior avaliação em comparação aos 
outros indicadores. $\mathrm{O}$ aspecto melhor avaliado foi a facilidade em marcar consulta com $22 \%$ de respostas "muito bom". A segunda pergunta mais bem avaliada foi a questão do apoio do pessoal do centro de saúde além do profissional que o atendeu com $22 \%$ de respostas "muito bom". Mesmo assim se analisarmos o percentual das respostas "regular", "ruim" e "péssimo" para as duas perguntas mencionadas, temos que $36 \%$ dos usuários entrevistados não encontram facilidade em marcar consulta e $28 \%$ dos entrevistados não consideram satisfatório o apoio do pessoal do centro de saúde (Gráfico 1).

A pior avaliação entre todas as variáveis componentes dos indicadores chave com a maior gradação das respostas negativas foi o tempo de espera para consulta: $56 \%$ dos usuários estavam insatisfeitos. Este foi o indicador chave com maior pro-

Tabela 1. Resultado de indicadores chaves de satisfação de usuários da ESF, Centro de Saúde Escola, Rio de Janeiro, 2010.

\begin{tabular}{lcc}
\hline \multicolumn{1}{c}{$\begin{array}{c}\text { Indice Bom/Muito Bom } \\
\text { por Indicador Chave }\end{array}$} & $\%$ & Classificação \\
\hline Relação e Comunicação & 88,9 & Muito Bom \\
Informação e Apoio & 82,3 & Bom \\
Cuidados Médicos & 79,5 & Bom \\
Continuidade e Cooperação & 61,0 & Regular \\
Organização do Serviço & 60,4 & Regular \\
\hline
\end{tabular}

Fonte: elaboração própria, 2011 porção de respostas "regular", "ruim" e "péssimo" em todas as perguntas.

Na ponderação de média global para os indicadores chave apresentado na Tabela 1 , o indicador chave que apresentou o melhor índice foi o de Relação e Comunicação, o único classificado como muito bom e os piores resultados dos indicadores chave foram Continuidade e Cooperação e Organização do Serviço ambos classificados como regulares.

Utilizando-se dos valores dos cinco Indicadores Chaves foi possível obter um Índice Global de Satisfação. Este índice constitui-se da média ponderada entre os Indicadores Chave. A média é ponderada pelo número de perguntas de cada indicador que não é constante. O índice global de satisfação no presente estudo foi de 76,6\%.

A análise de possíveis associações entre os padrões de resposta e as características dos usuários, a população com menor nível de escolaridade se mostrou mais satisfeita. Pessoas com boa autopercepção de saúde tendem a estar mais satisfeitas com os serviços prestados, e, os usuários mais jovens tendem a ser menos satisfeitos.

Por fim, uma vez que temos todas as respostas dos usuários segregadas por categorias (sexo, escolaridade, auto percepção de saúde e idade) podemos calcular os Índice Global específico de cada uma dessas categorias a fim de avaliar se estes índices são diferentes do já calculado.

O Gráfico 2, mostra as diferenças entre o índice Global de cada categoria e o índice Global da

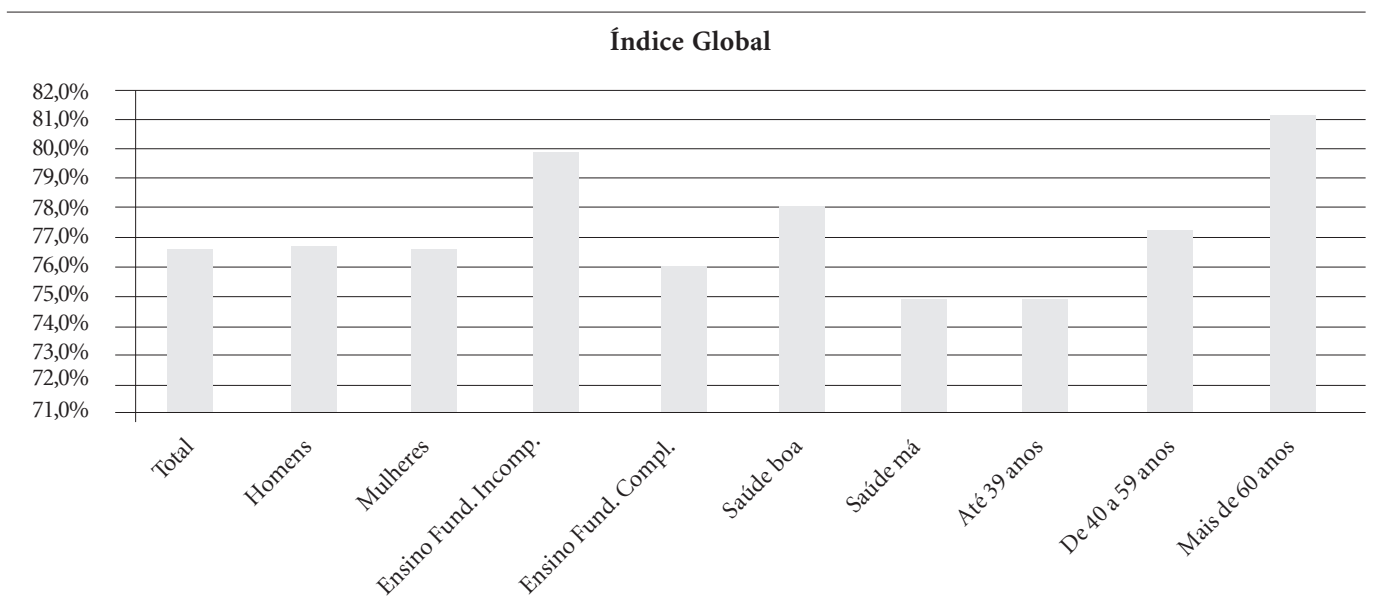

Gráfico 2. Índice Global Segundo Sexo, Nível de Escolaridade, Auto-Percepção de Saúde e Faixa Etária, ESF, Centro de Saúde Escola, Rio de Janeiro, 2010. 
população como um todo. Nele pode-se observar que o Índice de Satisfação Global de pessoas com auto percepção de saúde ruim é em torno de 1,6 pontos percentuais menor que o da população como um todo. Nota-se também que não há diferenças entre os Índices de Satisfação Global por sexo. Quanto à escolaridade, a população menos instruída tem maior Índice Global em relação ao total (79\%) enquanto que com a mais instruída ocorre o oposto (74\%). Vale também chamar atenção para a diferença deste índice no caso dos mais idosos (81\%).

Para facilitar a análise das sugestões e reclamações dos usuários da ESF, as categorias foram agrupadas em três blocos temáticos (Tabela 2). O bloco que obteve o maior número de menções dos usuários foi o relacionado à questão do acesso oportuno e o tempo de espera. Nessa categoria foram agrupadas questões como tempo para conseguir uma consulta, necessidade de maior agilidade do laboratório, pontualidade dos profissionais e das consultas e ampliação do horário de atendimento do Centro de Saúde e do laboratório. Essa categoria obteve 36\% (91) das menções.

O segundo bloco temático que recebeu maior número de reclamações/sugestões foi o relacionado à ampliação da oferta de profissionais e serviços com 32\% (82) das menções.

O outro bloco englobou as reclamações/sugestões dos usuários referente à organização do fluxo para atendimento no Centro de Saúde e atenciosidade com $28 \%$ (71) das sugestões. Neste item os usuários destacaram principalmente questões de atenção e respeito por parte dos profissionais do centro de saúde, limpeza, organização administrativa, priorização no atendimento, conforto e privacidade durante a consulta.

Tabela 2. Sugestões e reclamações dos usuários da ESF, Centro de Saúde Escola, Rio de Janeiro, 2010.

Sugestões e reclamações dos usuários

Acesso oportuno: tempo de espera, pontualidade

Acesso a consultas, tempo de espera para conseguir uma consulta

Maior rapidez e agilidade no atendimento do laboratório e na entrega do resultado

dos exames e ampliação do horário de funcionamento

Ampliação do horário de atendimento do centro de saúde

Pontualidade dos profissionais com o trabalho, faltas e greves

Pontualidade do atendimento de consultas marcadas

Sub total

Ampliação da oferta: de profissionais e serviços

Contratação de mais médicos

Contratação de especialidades

Maior número de visitas domiciliares

Trabalho do ACS, aumentar a realização de visitas domiciliares dos ACS

Ampliação da oferta de exames

Implantar serviço de urgência / emergência

Contratação de profissionais de Saúde da Família

Contratação de mais Dentistas

Farmácia, disponibilidade de medicamentos

Sub total

Organização dos fluxos no Centro de Saúde e atenciosidade

Organização do fluxo para atendimento no Centro de Saúde

Maior atenciosidade e respeito dos profissionais

Melhorar a limpeza do centro de saúde e banheiros

Priorização de atendimento de casos mais urgentes, crianças, gestantes e idosos

Conforto do Centro de Saúde

Maior privacidade no atendimento e das informações

Sub total

Outros

Total

Fonte: elaboração própria, 2011 


\section{Discussão}

O perfil observado na amostra de usuários do Centro de Saúde é similar àqueles de outros estudos de usuários da Atenção Básica ${ }^{12,13}$. A maior demanda nos serviços de saúde, em particular na Saúde da Família é de pessoas do sexo feminino, indicando que as mulheres utilizam os serviços de saúde com mais frequência, além de assumirem a responsabilidade em procurar atendimento para seus familiares e/ou amigos na sua comunidade.

Um fator que contribui para maior utilização dos serviços de saúde por mulheres é a fisiologia e os ciclos de vida das mulheres ${ }^{14,15}$. Além disso, os serviços e atividades de atenção primária foram tradicionalmente direcionadas ao grupo materno infantil e também na ESF são ofertadas diversas ações para este grupo prioritário, tais como: saúde da mulher, pré-natal, prevenção ao câncer ginecológico e de mama e saúde da criança.

Mais da metade dos entrevistados não possuía o ensino fundamental completo o que está de acordo com a literatura. Isto pode ser explicado pela instalação das unidades de saúde da família em regiões de baixa renda, com isso a escolaridade de usuários da ESF em geral é baixa. A população com um menor grau de escolaridade tende a emitir menos juízos de valor e ser mais condescendente com os serviços de saúde que lhe são prestados expressando graus mais elevados de satisfação, assim como foi observado no presente estudo ${ }^{12,15,16}$.

Em relação à faixa etária dos usuários entrevistados, a idade média foi em torno dos 40 anos, com destaque para faixas etárias mais elevadas, o que pode influenciar os resultados de satisfação, pois no presente estudo os idosos tenderam a ser mais satisfeitos com os serviços do que os jovens. Segundo Mishima et al. ${ }^{11}$ para os mais idosos a utilização desses serviços pode ter significado a busca para satisfação de outras necessidades, e não apenas a resolução de um problema de saúde, expresso em um agravo ou conjunto de sinais e sintomas, levando assim a uma maior satisfação.

A autoavaliação em saúde é um indicador mundialmente empregado para avaliar o estado de saúde $\mathrm{e}^{17}$ hoje esse indicador pode ser obtido em inquéritos nacionais no Brasil, como nas PNAD de 1998 e 2003. No ano de 2003, 21\% da população brasileira classificou sua saúde como regular, $4 \%$ classificou como ruim e $1 \%$ como muito ruim ${ }^{18}$.

A população de usuários do Centro de Saúde Escola caracterizou- se com pior percepção de saúde do que as médias obtidas em pesquisas nacionais, $45,8 \%$ dos usuários indicaram que sua saúde estava regular, esses são usuários que estão buscando o serviço de saúde, logo se encontram com alguma necessidade e demanda o que pode justificar essa pior autoavaliação do estado de saúde.

Em relação aos indicadores chave, a dimensão melhor avaliada nos estudos da ESF é a relacional, isto é, a relação entre usuário e profissional. No presente estudo também foi a melhor avaliada. É reconhecido na literatura que a produção da saúde demanda o estabelecimento de relações de intersubjetividade entre profissionais e usuários, abrindo-se a possibilidade de aproximação ao mundo da vida dos pacientes, portanto ao seu modo de adoecer e morrer ${ }^{19}$.

Com relação ao acesso aos serviços de saúde, a APS, como serviço de primeiro contato, articulado a outros níveis, pressupõe a constituição de uma rede integrada de serviços de saúde, a qual funciona como porta de entrada no sistema para o atendimento, ao longo do tempo, às necessidades e problemas de saúde das pessoas (não direcionados apenas à enfermidade), coordenando ou integrando os outros tipos de atenção, fornecidos em algum ponto do sistema de saúde.

A oferta de serviços e sua distribuição geográfica, a disponibilidade e a qualidade dos recursos humanos e tecnológicos, os mecanismos de financiamento, o modelo assistencial e a informação sobre o sistema são características da oferta que afetam o acesso ${ }^{12}$. Segundo Van Stralen et al..$^{19}$ a dimensão do acesso diz respeito principalmente à maior ou menor facilidade da população para obter uma consulta. Ainda que não devamos reduzir a atenção primária à prestação de consultas médicas, esta atenção não pode abdicar da atenção clínica prestada pelos profissionais de saúde.

No presente estudo foram observadas dificuldades relacionadas ao acesso, principalmente para se conseguir consultas e serviços de apoio diagnóstico e terapêutico. Esses fatores constituem-se como grande desafio, na busca da integralidade da atenção ${ }^{20}$.

A organização dos serviços foi o indicador pior avaliado e foi uma das dimensões mais mencionadas como sugestão/reclamação na pergunta aberta presente no questionário.

Em estudo que avaliou a satisfação de usuários em dois municípios de Pernambuco, constata-se um predomínio de fatores ligados à organização dos serviços como causas para as dificuldades no acesso aos cuidados de saúde ${ }^{21}$. 
A dimensão de Cuidados Médicos recebidos é menos abordada na literatura em geral. No presente estudo essa dimensão foi avaliada como boa, com destaque para o exame físico que o profissional realizou e a melhora que o atendimento proporcionou para o desempenho e realização das tarefas diárias. Segundo Trad et al. ${ }^{13}$, ainda que de forma fragmentada, os usuários do PSF identificam que as equipes realizam um tipo de cuidado que diferenciado em relação a práticas de atividades de promoção e prevenção.

$\mathrm{Na}$ dimensão de Informação e Apoio, os itens que mais se destacaram positivamente, foi a explicação do profissional sobre exames e informações sobre os sintomas da doença. No estudo de avaliação da qualidade do PSF no Ceará segundo os usuários a dimensão de informação foi considerada satisfatória. Apenas 14\% dos usuários afirmaram sair da consulta com dúvi$\mathrm{da}^{15}$. Também, no estudo que avaliou a satisfação dos usuários com a ESF em município de Minas Gerais, 96,2\% dos usuários estavam satisfeitos com a clareza nas explicações realizadas aos pacientes ${ }^{16}$.

A abordagem de questões emocionais mencionada no indicador informação e comunicação é essencial para a avaliação da qualidade de vida do paciente, assim como identificar fatores externos eventualmente relacionados ao problema apresentado. É uma abordagem que deve fazer parte da relação médico-paciente, de modo a permitir a prática de um cuidado integral à saúde.

Em relação ao indicador continuidade e cooperação o conhecimento do profissional em relação ao usuário pode ter sido facilitado pela avaliação de usuários atendidos na unidade de saúde em período superior a um ano (critério de inclusão no estudo), o que permite maior conhecimento do caso, e fortalecimento da continuidade do cuidado ao paciente. Além disso, reforça-se aqui a importância para a avaliação da continuidade, a existência de meios de registro de informações eficazes, de modo a permitir que todos os dados sejam corretamente registrados em prontuários, e devidamente acessados, quando necessário.

Observa-se concordância entre os achados provenientes da análise quantitativa e da análise qualitativa das variáveis do instrumento utilizado. $\mathrm{Na}$ análise dos indicadores chaves, o pior indicador, classificado como regular, foi o da organização do serviço de saúde e de acordo com as sugestões/reclamações apontadas na pergunta aberta, esse bloco foi um dos mais mencionados, além das questões como acesso, tempo de espera e a organização do fluxo para atendimento no Centro de Saúde Escola.

Vale ressaltar que as sugestões dos usuários de melhorias propostas para a unidade são em sua maioria bastante factíveis. Não demandam grandes investimentos financeiros e sim iniciativas de reorganização dos processos de trabalho, assim como otimização dos fluxos internos, mostrando que a avaliação dos usuários pode contribuir para melhorar os problemas do cotidiano dos serviços de saúde.

De acordo com documentos oficiais do EUROPEP o instrumento utiliza o conceito de "avaliação dos pacientes”, referindo-se a avaliações subjetivas dos diferentes aspectos da prestação do cuidado em relação a pontos positivos e negativos do cuidado recebido ${ }^{22}$. Considerando que a avaliação é igual à expectativa em relação ao cuidado menos a experiência do cuidado recebido. Justamente por isso solicitou-se aos entrevistados avaliações diretas ${ }^{11}$.

A avaliação dos usuários foi em geral positiva, pode-se dizer mais do que o esperado, dada as condições de difícil acesso no Rio de Janeiro, e isto requer algumas considerações no que se refere a forte relação entre expectativa e satisfação. Quando a satisfação é menor que a expectativa, menor será a satisfação e vice versa, porém quando as expectativas são baixas mais facilmente são atingidas e um nível alto de satisfação será atingido, ou seja, quanto maior for a discrepância entre as expectativas e a experiência do cuidado, maior será a satisfação ou insatisfação com o serviço de saúde.

Os achados de alta satisfação no presente estudo podem estar relacionados a um nível baixo de expectativas, já que a rede de serviços de saúde no Rio de Janeiro configura-se como uma rede com dificuldades de acesso pelos usuários. Com isso a expectativa em relação aos serviços consequentemente é baixa ${ }^{19}$. De acordo com a relação exposta acima, com expectativa baixa mais facilmente se alcança a satisfação, mesmo quando a qualidade do serviço prestado não é tão elevada assim.

A alta satisfação apresentada em estudos como esses, segundo Melo et al. ${ }^{18}$, pode ser explicada também por conta das relações assimétricas (usuários/profissionais/gestores) e não participativas, que pouco contribuem para o desenvolvimento da capacidade crítica e reinvidicativa dos atores sociais envolvidos.

Autores como Costa et al. ${ }^{22}$, enfatizam que há certa "naturalização" para baixa qualidade na visão dos usuários os quais terminam por reco- 
nhecer o serviço recebido como um favor ou doação e não como um direito, o que representa uma das diferentes interpretações para o gratuito.

É necessário observar que um dos limites de metodologias como essa, com aplicação de questionário logo após a consulta, podem induzir ao viés da gratidão, principalmente ao se tratar de um serviço público que pode gerar receio nos usuários em perder o acesso ao serviço.

Outras considerações devem também ser feitas em relação à aplicação e adaptação do instrumento. Como outra limitação do método realizado, destaca-se que o instrumento foi adaptado de uma versão europeia para uma realidade de grande centro urbano da região sudeste do Brasil. Pode não ser adequado para todo o país, onde estão em jogo outras realidades (econômicas, sociais, demográficas, etc.), sendo necessárias outras adaptações. Além disso, o instrumento foi idealizado para avaliar a atuação do profissional médico de família e como a ESF é composta por equipe multiprofissional, é necessário complementar o instrumento com perguntas específicas aos outros profissionais.

Aspectos metodológicos que merecem ainda destaque são as formas de abordagem e seleção dos usuários, o local da realização das entrevistas no fluxo de saída, nem sempre tendo sido possível selecionar os usuários de forma sistemática, o que é uma dificuldade sempre encontrada por este tipo de abordagem.

Outra distinção está em que na Europa utiliza-se o instrumento de forma autoaplicável, onde as pessoas recebem o questionário e deve devolver por correio com um selo para o envio do questionário preenchido para o centro de saúde. No processo de adaptação, o instrumento foi aplicado por entrevistadores treinados e a aplicação foi realizada do lado de fora do Centro de Saúde após o atendimento do usuário, o que pode produzir vieses.

\section{Considerações finais}

Considera-se que os processos de avaliação da satisfação dos usuários nos serviços públicos de saúde podem atuar para a construção de uma nova perspectiva do cuidado e que, além de monitorar as atividades desses serviços, pode fortalecer o controle social e a participação/inclusão dos usuários nos processos de planejamento. Pode contribuir portanto, para a construção de alternativas conjuntas - entre serviços de saúde e usuários - para intervenções mais adequadas para solucionar problemas presentes no cotidiano dos serviços, propiciando avanços no âmbito da produção de cuidados e gestão dos serviços de saúde.

A avaliação da satisfação dos usuários dos serviços de saúde é ferramenta importante para subsidiar o processo de decisão compartilhada, de forma a se repensar as práticas profissionais, reorganizar o processo de trabalho desenvolvido, realocar recursos, readequar ações e redefinir objetivos que estejam coerentes com o projeto de saúde estabelecido.

No presente estudo os resultados que se destacam são: a boa avaliação da relação entre usuários e profissionais e as críticas em relação à organização dos serviços e aos tempos de espera para consultas. Os resultados encontrados se aproximaram de vários outros estudos de avaliação da ESF, como por exemplo, na parte correspondente à avaliação de satisfação na pesquisa mundial de saúde, o critério que obteve os piores graus foi o tempo de espera para atendimento, enquanto aspectos como intimidade, o sigilo das informações pessoais e as competências dos profissionais de saúde receberam a pontuação mais elevada de satisfação ${ }^{23}$.

Os resultados deste estudo apontam que para a comparação entre unidades é necessário a composição de amostras com grupos etários e de auto percepção de saúde similares para possibilitar a comparações entre diferentes unidades de saúde e municípios.

O instrumento possui como pontos positivos, o fato de ser um questionário simples, de rápida aplicação e de fácil preenchimento, com possibilidades de reaplicabilidade da metodologia. Podendo ser aplicado rotineiramente como prática dos processos de trabalho dos serviços de saúde, demonstrando assim utilidade prática para a gestão da atenção primária à saúde, favorecendo processos avaliativos de natureza interna (autoavaliação) e externa e configurando-se como um instrumento de institucionalização da avaliação desde a perspectiva dos usuários.

O presente trabalho foi capaz de demonstrar a importância de se avaliar a satisfação do cuidado sob a perspectiva do usuário, principalmente pela aplicabilidade prática do instrumento de avaliação desenvolvido, e a factibilidade das sugestões provenientes da avaliação dos usuários, representando importante ferramenta para a institucionalização da avaliação na ESF. 


\section{Colaboradores}

ALRBS Brandão - concepção, delineamento, análise e interpretação dos dados e redação do artigo. L Giovanella - concepção, redação do artigo e revisão crítica. CEA Campos - concepção e revisão crítica.

\section{Referências}

1. Conill EM. Ensaio histórico-conceitual sobre a Atenção Primária à Saúde: desafios para a organização de serviços básicos e da Estratégia Saúde da Família em centros urbanos no Brasil. Cad Saude Publica 2008; 24(Supl. 1):7-27.

2. Felisberto E. Da teoria à formulação de uma Política Nacional de Avaliação em Saúde: reabrindo o debate. Cien Saude Colet 2006; 11(3):553-563.

3. Esperidião M, Trad LAB. Avaliação da satisfação de usuários. Cien Saude Colet 2005 (Supl. 10):303-312.

4. Crow R, Gage H, Hampson S, Hart J, Kimber A, Storey L, Thomas H. The measurement of satisfaction with healthcare: implications for practice from a systematic review of the literature. Health Technol Assess 2002; 6(32):1-10.

5. Ferreira PL, Raposo V. A governação em saúde e a utilização de indicadores de satisfação. Rev Port Clin Geral 2006; 22:285-296.

6. Esperidião MA, Trad LAB. Avaliação de satisfação de usuários: considerações teórico-conceituais. Cad Saude Publica 2006; 22(6):1267-1276.

7. Silva A. A framework for measuring responsiveness. Geneva: WHO; 1999. (GPE Discussion Paper series, n. 32)

8. Albuquerque AB, Deveza M. Adesão ao tratamento na prática do Médico de Família e Comunidade e na Atenção Primária à Saúde. Porto Alegre: Promef-Artmed 2009; 3(4):41-72.

9. Kloetzel K, Bertoni AM, Irazoqui MC, Campos VPG, Santos RN. Controle de qualidade em atenção primária à saúde. I - A satisfação do usuário. Cad Saude Publica 1998; 14(3):623-628.

10. Grol R, Wensing M. Patients evaluate general/family pratice. The EUROPEP Instrument. $\mathrm{Br} J \mathrm{Gen}$ Pract 2000; 50:882-887.

11. Mishima SM, Pereira FH, Matumoto S, Fortuna CM, Fortuna CM, Pereira MJB, Campos AC, Paula VG, Domingos MMLN. A assistência na saúde da família sob a perspectiva dos usuários. Rev Lat Am Enfermagem 2010; 18(3):148-155.

12. Ibañez N, Rocha JSY, Castro PC, Ribeiro MCSA, Forster AC, Novaes MHD, Viana ALDA. Avaliação do desempenho da atenção básica no Estado de São Paulo. Cien Saude Colet 2006; 11(3):683-703.

13. Trad LAB, Bastos ACS, Santana EM, Nunes M. O estudo etnográfico da satisfação de usuário do Programa de Saúde da Família (PSF) na Bahia. Cien Saude Colet 2002; 7(3):581-589.

14. Jorge MSB, Guimarães JMX, Vieira LB, Paiva FDS, Silva DR, Pinto AGA. Avaliação da qualidade do Programa de Saúde da Família no Ceará: a satisfação dos usuários. Rev. Baiana de Saúde Pública 2007; 31(2):256-266.

15. Cotta RMM, Marques E S, Maia TM, Azeredo CM, Schott M, Franceschini SCC, Priore SEA. Satisfação dos usuários do Programa de Saúde da Família: avaliando o cuidado em saúde. Scientia Medica 2005; 15(4):227-234

16. Barata RB. Condições de Saúde da População Brasileira. In: Giovanella L, Escorel S, Lobato LVC, Carvalho AI, Noronha JC, organizadores. Políticas e Sistema de Saúde no Brasil. 1a Edição. Rio de Janeiro: Fiocruz; 2008. p. 173:175. 
17. Instituto Brasileiro de Geografia e Estatística (IBGE). Pesquisas Nacionais por Amostra de Domicílios. Rio de Janeiro: IBGE; 2003.

18. Melo EM, Paiva L, Álvares J, Flecha ALD. A organização da atenção básica em municípios intergantes do Projeto de Expansão e Consolidação do Saúde da Família em Mato Grosso, Brasil. Cad Saude Publica 2008; 24(Supl. 1):29-41.

19. Van Stralen CJ, Belisário SA, Van Stralen TBD, Lima AMD, Masote AW, Oliveira CL. Percepção dos usuá rios e profissionais de saúde sobre a atenção básica: comparação entre unidades com e sem saúde da família na Região Centro-Oeste do Brasil. Cad Saude Publica 2008; 24(Supl. 1):148-158.

20. Samico I, Hartz MZA, Felisberto E, Carvalho EF. Atencão à saúde da criança: uma análise do grau de implantacão e da satisfacão de profissionais e usuários em dois municípios do estado de Pernambuco. Revista Brasileira de Saúde Materno Infantil 2005; 5(2):229-240.

21. Jung H, Van HF, Wensing M, Hearnshaw H, Grol R. Which aspects of general practitioners' behavior determine patients' evaluations of care? Soc Sci Med 1999; 47(8):1077-1087.

22. Costa GD, Cotta RMM, Franceschini SCC, Batista RS, Gomes AP, Martins PC, Ferreira MLSM. Avaliação em saúde: reflexões inscritas no paradigma sanitário contemporâneo. Physis 2008; 18(4):705-726.

23. Gouveia GC, Souza WV, Luna CF, Junior PRBS, Szwarcwald CL. Health care user's satisfaction in Brazil, 2003. Cad Saude Pública 2005; (Supl. 21):109-118.

Apresentado em 02/03/2012

Aprovado em 09/04/2012

Versão final apresentada em 28/06/2012 